\title{
Civilisations
}

Revue internationale d'anthropologie et de sciences

humaines

58-1 | 2009

American Afrocentrism(e)s américains

\section{Africain, Akan, Panafricain et Afro-Américain}

Construire son identité aux États-Unis

Pauline GUEDJ

\section{(2) OpenEdition}

Journals

Édition électronique

URL : http://journals.openedition.org/civilisations/1925

DOI : $10.4000 /$ civilisations. 1925

ISSN : 2032-0442

Éditeur

Institut de sociologie de l'Université Libre de Bruxelles

Édition imprimée

Date de publication : 31 août 2009

Pagination : 73-92

ISBN : 2-87263-026-0

ISSN : 0009-8140

\section{Référence électronique}

Pauline GUEDJ, « Africain, Akan, Panafricain et Afro-Américain », Civilisations [En ligne], 58-1 | 2009, mis en ligne le 31 août 2012, consulté le 01 mai 2019. URL : http://journals.openedition.org/ civilisations/1925; DOI : 10.4000/civilisations.1925 


\title{
Identités afrocentristes entre religion et politique
}

\author{
Africain, Akan, Panafricain et Afro-Américain \\ Construire son identité aux Etats-Unis
}

\author{
Pauline GUEDJ
}

Résumé : Fondé dans les années 1960, le mouvement " akan " rassemble plusieurs milliers de fidèles. Né de la rencontre d'un percussionniste afro-américain new-yorkais et de la prêtresse en chef d'un sanctuaire ghanéen, il s'appuie sur la reproduction en Amérique du Nord du culte de divinités akan et instaure des liens généalogiques entre Akan du Ghana et Afro-Américains descendants d'esclaves. En revenant sur la formation et sur le fonctionnement actuel du mouvement, cet article s'intéresse aux relations observables entre constructions identitaires et pratiques rituelles. A travers l'analyse des multiples espaces rituels du culte " akan » américain, il démontre comment la tentative d'invention de l'Afrique opérée par les fidèles s'accompagne de logiques d'identification complexes, leur permettant de se revendiquer tantôt akan, tantôt panafricains, et afro-américains.

Mots-clés : Afro-Américains, Etats-Unis, Akan, Panafricanisme, religion, identité.

Abstract: Founded in the 1960s, the "Akan " movement now has thousands of members in the US. Born from the encounter of an Afro-American musician and the chief priestress of a Ghanaian shrine, it reproduces the worship of Akan divinities in North America, and institutes genealogical links between Akan people from Ghana and Afro-Americans descendants of slaves. This paper describes the development and the current dynamics of the movement, and highlights the relationships between identities and ritual practices. The article also describes the multiple ritual spaces of the American "Akan" movement, and shows how, for Afro-American "Akans", the invention of Africa goes along with complex logics of identification, which allow the members to position themselves simultaneously as Akan, Panafrican, and Afro-Americans.

Key words: Afro-Americans, United States of America, Akan, Pan-africanism, Religion, Identity. 
$\mathrm{L}$ 24 janvier 1965, Malcolm X, ancien porte-parole de la Nation of Islam ${ }^{1}$ et leader d'une organisation nouvellement créée, l'Organization of Afro-American Unity, prononce un célèbre discours où il revient sur les catégories nominales utilisées aux Etats-Unis pour désigner les populations afro-américaines ${ }^{2}$. Malcolm X est assassiné à peine un mois plus tard mais son discours sera publié par son épouse Betty Shabazz en 1967 sous le titre Malcolm X on Afro-American History.

Dans ce texte, Malcolm X s'exclame : « Un des objectifs de notre appellation Negro est de nous faire oublier qui nous sommes réellement. Le terme Negro ne veut rien dire. Il ne vous réfère pas à une langue parce que la langue Negro n'existe pas. Il ne vous donne pas un pays parce que le pays Negro n'existe pas. Il ne vous rattache pas à une culture parce que la culture Negro n'existe pas non plus. La terre, la culture, la langue Negro n'existent pas et l'homme Negro n'existe pas non plus » (1967: 7).

Pour Malcolm X, le Noir américain souffre aux Etats-Unis d'un état d'anomie. Victime d'une entreprise de «lavage de cerveau $»^{3}$ menée par les Blancs, l'homme noir en est arrivé à perdre toute conscience de ses origines, de son Histoire. Afin de pallier cette crise identitaire, il doit refaire corps avec sa culture originelle, recouvrer son identité première bafouée et vivre conformément à son essence, à ses déterminismes culturels et biologiques. L'homme noir doit donc revendiquer sa spécificité sur la scène nationale, connaître son passé pour mieux construire son futur et justifier ainsi ses apports culturels dans une Amérique, terre de la diversité et de la singularité plurielle.

Par conséquent, chez Malcolm X, le terme Negro devient un symbole du supposé état d'anomie des populations afro-américaines. C'est un emblème de cet oubli forcé des origines dont souffre tout Noir américain. « Nous ne sommes pas des Negroes, ditil plus loin. Nous n'avons jamais été des Negroes avant d'être transportés ici et d'être transformés en cela. Nous avons été créés scientifiquement par l'homme blanc. Celui qui se définit comme Negro est un produit de la civilisation occidentale ; et pire encore d'un crime occidental » (1967: 8).

Le terme Negro renverrait donc à une société blanche esclavagiste qui a volontairement contraint les populations noires à l'oubli de soi et à la perte des repères culturels. Se détacher du terme Negro reviendrait alors à s'émanciper d'une posture de victimes, d'asservis pour endosser une nouvelle identité liée à un passé imaginé comme glorieux voire supérieur. Chez Malcolm X, comme ce sera le cas chez de nombreux militants du nationalisme noir, rejeter le terme Negro c'est alors nier la rupture fondatrice causée par la tragédie de l'esclavage. La continuité dans la discontinuité, comme disait Roger Bastide (1996) lorsqu'il évoquait le dilemme des constructions identitaires des représentants

1 La Nation of Islam est un mouvement nationaliste Afro-Américain né dans les années trente. Ses leaders prônent le retour des Afro-Américains à ce qu'ils considèrent comme leur religion originelle, l'islam, et réclament l'obtention d'un Etat indépendant sur le territoire états-unien. Sur la Nation of Islam voir, entre autres, Brent Turner (1984), Essien-Udom (1964), Gardell (1996).

2 J'utilise dans ce texte le terme « Afro-Américain » pour qualifier les populations noires des Etats-Unis. Majoritairement mobilisé dans les écrits anthropologiques sur les Amériques noires, ce terme a l'avantage de ne pas renvoyer aux débats idéologiques liés aux catégories de désignation des Noirs aux Etats-Unis.

3 L'expression « lavage de cerveau » est utilisée au sein de la Nation of Islam pour dénoncer les actions d'une population blanche qui chercherait à faire oublier aux Afro-Américains leur réelle identité. 
de ses Amériques Noires, est ainsi instaurée et l'Histoire rattacherait sans rupture les populations noires des Etats-Unis à celles du continent africain.

Aujourd'hui, cette idée de rattacher consciemment les populations afro-américaines à leur Histoire d'avant la traite négrière se retrouve dans les écrits des penseurs afrocentristes. Molefi Asante, chef de file de cette mouvance, considère lui aussi que les populations noires doivent refaire corps avec leur identité ancestrale pour vivre librement aux Etats-Unis ${ }^{4}$. L'Afrocentricité, théorie dont il ne cesse de retravailler la définition, devient alors un ensemble de " règles de vie » dont l'adoption aurait en elle-même un pouvoir performatif. Etre Afrocentriste, c'est adhérer à ce mode de vie idéal et par ce fait même revigorer ses racines, recouvrer son essence. L' "Africain authentique » est l'Afrocentriste, puisque lui seul est à même de reconquérir, par ses actes, une Afrique ancestrale dont l'Histoire serait exempte des méfaits d'une civilisation occidentale colonialiste et esclavagiste.

En 2005, ce pouvoir performatif de l'afrocentricité est rappelé par Asante lors d'une communication délivrée à un colloque intitulé « Le rôle de l'afrocentrictité dans la renaissance africaine » organisé les 29 et 30 juillet à Cotonou au Bénin. Dans son allocution $^{5}$, l'auteur insiste sur la « crise culturelle » dont souffriraient Africains et AfroAméricains et sur la nécessité, pour tout Noir, d'amorcer une entreprise de retour aux sources qu'il nomme la « résurgence africaine ». Or, pour Asante, c’est en Egypte, dans la terre noire de Kemet, que l'Africain et l'Afro-Américain doivent chercher la forme embryonnaire de cette Afrique originelle à recréer et à recouvrer. « Kemet est la meilleure source à laquelle nous puissions puiser pour notre renaissance, car la position historique de Kemet est indéniable » écrit-il. «C'est de là que l'énergie qui a assuré l'expansion d'idées africaines importantes a irradié. De la même façon que la Chine et l'Inde sont à la source des cultures de l'Asie, de la même façon que la Grèce et Rome sont à la source des cultures de l'Europe, Kemet et la Nubie sont à l'origine de bien des idées africaines [...] Etant donné le système culturel dont nous, les Africains, avons hérité de l'Europe colonisatrice, une force historique nous pousse à retourner à l'identité qui était la nôtre avant que nous soyons asservis, afin de nous renouveler. L'esclavage ne peut pas nous revitaliser, il nous faut nous tourner vers la période qui a précédé l'esclavage » (Asante $2005)^{6}$.

Chez Asante, cette « période qui a précédé l'esclavage » s'incarne dans un fantasme de l'Egypte antique, comme source d'une culture africaine imaginée comme unique et unifiée. L'Egypte, si fréquemment évoquée dans les écrits des Afrocentristes, se fait alors le symbole d'un passé glorieux prouvant non seulement l'absence de rupture dans

4 Dans son ouvrage L'Atlantique noir (2003), Paul Gilroy revient sur cette tentative d'outrepasser le traumatisme de l'esclavage dans le projet afrocentriste. «Elan », selon ses propres termes, « révisionniste », l'oubli programmé de l'esclavage, son « refoulement » vont de pair avec la construction d'une tradition perçue comme immuable, « faisant de la pureté la base de la solidarité raciale » (Gilroy 2003 : 248-249).

5 La communication de Molefi Asante fut en réalité lue à l'audience, Asante étant absent de la conférence.

6 Texte disponible sur : http://www.africamaat.com/L-Afrocentricite-et-la-Resurgence, consulté le 29 janvier 2009. 
l' « expérience » des populations noires de la planète mais également leur supériorité, leur « génie », responsable de la plus noble civilisation de l'Histoire humaine?.

Depuis les années 1930, plusieurs civilisations, plusieurs pays ont alors été imaginés par les militants du nationalisme noir comme autant de terres d'origine du peuple afroaméricain. Pour les leaders de la Nation of Islam, La Mecque n'est autre que le lieu de naissance de la Tribu de Shabazz, ancêtre des Noirs de l'actuelle Amérique, créée par Allah à son image. Pour les membres du mouvement " yoruba », le Nigeria et le Bénin, territoires des anciens empires et royaumes yoruba, font figure de terres ancestrales avec lesquelles se tissent des connexions transnationales et des réseaux lignagers ${ }^{8}$. Enfin, pour les pratiquants de la religion « akan », le Ghana, nation pétrie de panafricanisme, constitue le pays phare d'une entreprise de quête des origines faite d'allées et venues entre les deux continents et de périples initiatiques ${ }^{9}$.

L'objectif de cet article est d'analyser les stratégies mises en œuvre par les AfroAméricains pour reconstituer aux Etats-Unis un passé, une mémoire, qui comme l'a souligné Maurice Halbwachs (2002), se pratique à partir d'enjeux contemporains ${ }^{10}$. En revenant sur une série d'enquêtes ethnographiques réalisées entre 2000 et 2007 au sein du mouvement «akan » américain, je montrerai comment ce passé réinventé s'appuie sur des constructions identitaires complexes et comment il se met en scène dans l'action rituelle. Ce faisant, nous verrons comment l'invention d'une tradition " akan » aux Etats-Unis s'élabore autour de nombreuses catégories d'identitification qui se mêlent et s'entrelacent. Tantôt Akan, tantôt panafricains et tantôt Afro-Américains, les fidèles du mouvement construisent leur identité et multiplient les espaces du rituel ${ }^{11}$. L'esclavage, tant occulté par les Afrocentristes, se trouvera alors repensé et réincorporé.

\section{L'histoire du mouvement « akan "}

Le mouvement «akan » est né en 1965 lorsque Gus Edwards, percussionniste afroaméricain et leader d'une compagnie de danse, partit en tournée en Afrique de l'Ouest. Depuis plusieurs années, Edwards, militant du nationalisme culturel, était impliqué dans la valorisation de l'héritage africain des Noirs américains. Dans les années 1940, il avait fondé une association, The Ghanas dont l'objectif était d'organiser des actions culturelles - expositions sur l'Afrique et les populations noires, cours de danse et de percussions - destinées exclusivement à une audience afro-américaine. En 1955, il avait été à l'origine d'une " société secrète », Damballah Hwedo dont les membres cherchaient à reproduire aux Etats-Unis les pratiques rituelles des Yoruba du Nigeria, des Akan du Ghana et du vodou haïtien. Au début des années 1960, après s'être rebaptisé Dinizulu en

7 L'Egypte antique constitue un motif fréquemment utilisé par les militants du nationalisme noir. Wilson Jeremiah Moses (1998) appelle « contributionnisme », la posture adoptée par Asante qui suppose l'utilisation de la civilisation égyptienne pour prouver le rôle des Africains dans l'Histoire de l'humanité. Voir aussi Walker (2004).

8 Sur le mouvement « yoruba », voir Capone (2005), Clarke (2004) et Palmié (1995).

9 Sur les «Akan », voir Guedj (2006). Dans cet article, les termes akan et yoruba seront placés entre guillemets lorsqu'ils sont relatifs aux mouvements états-uniens, pour les distinguer des ethnies africaines.

10 Les références sur les constructions sociales de la «tradition » et de la « mémoire » sont nombreuses. Voir également, Hobsbawm et Ranger (2006), Lenclud (1994) et Pouillon (1975).

11 Ce présent article reprend et complète des arguments développés dans Guedj (2005 et 2007). 
l'honneur d'un chef zoulou de l'actuelle Afrique du Sud, il créa sa compagnie de danse, The Dinizulu African Dancers, Singers and Drummers, et commença à rassembler une importante collection d'art africain et de documents historiques relatifs à la traite négrière.

En 1965, alors que la compagnie s'était produite dans de nombreux festivals étatsuniens, Dinizulu et ses danseurs s'envolèrent pour l'Afrique de l'Ouest où ils entamèrent une tournée de plusieurs semaines. La tournée mena les danseurs dans différents pays, au Nigeria et en Côte d'Ivoire en particulier, mais c'est au Ghana qu'ils séjournèrent le plus longtemps ${ }^{12}$. Au Ghana, Dinizulu visita d'abord Accra où il effectua des recherches sur les traditions ouest-africaines et akan à la bibliothèque de l'université de Legon. Il se rendit également dans plusieurs sites de la région tels l'Aburi Botanical Garden et le marché de Koforidua. Rapidement, ses pérégrinations le menèrent dans un sanctuaire de la religion dite « traditionnelle », l'Akonedi Shrine de Larteh Kubease, très populaire dans le pays du fait notamment de ses connexions avec le pouvoir politique ${ }^{13}$.

A Larteh Kubease, Dinizulu fut accueilli par Nana Ekua Oparebea, prêtresse en chef du lieu, qui décida de lui ouvrir les portes de son sanctuaire et de lui enseigner les rudiments des rituels qui y étaient perpétués. La prêtresse avait conscience que de nombreux esclaves déportés en Amérique du Nord étaient en réalité originaires du Ghana actuel. Elle proposa donc à l'Américain d'effectuer pour lui une séance de divination (reading) lors de laquelle elle serait, pensait-elle, à même de le renseigner sur les origines de ses ancêtres et sur la réalité de sa mission au Ghana et en Amérique ${ }^{14}$.

Pendant la séance de divination, Oparebea utilisa ses dons de médium pour communiquer avec les divinités (abosom). Celles-ci auraient alors révélé à l'Américain que ses ancêtres étaient originaires d'un village voisin, qu'ils appartenaient à la famille de la prêtresse elle-même et qu'ils étaient membres du groupe ethnique akan. Par ailleurs, Dinizulu reçut, lors de ce rituel, la mission de partir à la recherche de ces Afro-Américains, qui, comme lui, auraient des ancêtres akan et seraient désireux de (re)découvrir leurs origines ancestrales. Il se vit décerner un nouveau nom, Nana Yao Opare Dinizulu, reçut le titre d'Omanhene, " chef traditionnel des Akan d'Amérique » et obtint un premier enseignement sur le culte des abosom (sing. obosom), divinités vénérées au sanctuaire.

De retour aux Etats-Unis, Dinizulu continua à entretenir des rapports étroits avec Oparebea. Il honora sa mission en se lançant dans une véritable campagne de conversion visant à raviver les origines akan de ses concitoyens afro-américains. Deux ans plus tard, il loua un bâtiment situé au 115, 321 th Street à Long Island City dans le Queens de New York. C'est là qu'il fonda, le premier Temple « akan », le Bosum Dzemawodzi,

12 Le Ghana exerçait en effet à l'époque un réel pouvoir de fascination sur les militants afro-américains. Indépendant depuis 1957 , le pays était devenu un symbole dans la lutte des populations noires contre l'oppression. Nombreux furent les activistes qui s'inspirèrent de l'exemple ghanéen dans leur propre combat, à l'image de W.E.B. Du Bois qui mourut à Accra en 1963 après avoir obtenu la nationalité ghanéenne. Par ailleurs, le Ghana bénéficiait de la réputation de rebelle qui incombait souvent dans la littérature historique et anthropologique au peuple akan. Considérés comme les ancêtres des esclaves marrons, les Akan faisaient figure de population insoumise, caractère insoumis auquel s'associent aujourd'hui les membres du mouvement « akan » états-unien. Sur l'intérêt des Afro-Américains pour le Ghana, voir aussi la contribution de Sarah Fila-Bakabadio dans ce numéro.

13 Depuis 1962, Nana Oparebea, prêtresse en chef de l'Akonedi Shrine de Larteh Kubease, était à la tête de la Ghana Psychic and Traditional Healing Association, association fondée par Kwame Nkrumah pour sauvegarder la pratique de la médecine traditionnelle au Ghana.

14 Sur l'histoire de l'Akonedi Shrine, voir Brokensha (1966) et Guedj (2008). 
ainsi qu'un centre culturel nommé The Aims of the Modzawe ${ }^{15}$. Il inaugura le Temple en y célébrant l'odwiraa, "Nouvel an africain », symbolisant, dans les termes du leader, " l'héritage africain des Afro-Américains ». Plus tard, la même année, il organisa un mariage (aweregye) ainsi qu'une cérémonie de dation du nom à un nourrisson (edin toa). Plusieurs fois par semaine, Dinizulu proposait des cours sur l'histoire de l'Afrique et offrait à ses adhérents une formation en twi. Parallèlement, il institua, à l'intérieur du centre culturel, un musée destiné à rassembler les objets africains et les ouvrages sur le continent noir qu'il avait récoltés depuis une vingtaine d'années.

De son côté, Oparebea accueillit au Ghana de nombreux Afro-Américains, qui cherchaient eux aussi à redécouvrir leurs origines, et même pour certains, à se faire initier à la prêtrise. Entre 1971 et 1995, date de son décès, la prêtresse effectua neuf voyages en Amérique du Nord, à New York, Philadelphie et Washington en particulier. Au cours de ses séjours états-uniens, elle fonda de nombreuses maisons de culte, de sorte qu'à partir de la fin des années 1970, l'ensemble des lieux de culte « akan » formait un vaste mouvement, qui bien que fragilisé par des luttes internes, entretenait toujours des relations étroites avec la maison mère ghanéenne.

Aujourd'hui, le mouvement « akan » rassemble quelques milliers de fidèles. Chaque maison de culte est dirigée par un prêtre ou une prêtresse, que les adeptes appellent Nana. Les cérémonies et les rituels ont généralement lieu dans la demeure de cet officiant où l'on trouve une chambre destinée à rassembler les autels des divinités. Le (ou la) Nana est chargé(e) d'organiser les cérémonies, de nourrir les autels et de veiller à l'initiation des godchildren ou akomfowa, personnes destinées à la prêtrise. Dans ces tâches, il (ou elle) est assisté(e) par des prêtres secondaires, les akomfo. Les lieux de culte « akan » ouvrent également leurs portes aux adeptes dont la vocation n'est pas la pratique de la prêtrise ainsi qu'à des sympathisants qui participent aux cérémonies et sont clients des séances de divination (reading).

\section{Devenir «Akan » aux Etats-Unis}

A l'image des pionniers du mouvement « akan », la plupart des adeptes actuels de cette religion aux Etats-Unis ont intégré des maisons de culte pour assouvir leur soif de connaissance de l'Afrique. Ainsi, l'assimilation d'un Afro-Américain au mouvement est le résultat d'un long processus au cours duquel celui-ci s'est documenté sur les cultures de l'Afrique occidentale, a lu des ouvrages ou consulté des sites internet consacrés à la «Terre Mère », pris des cours de danse et/ou fréquenté des associations organisant des conférences et des expositions. Rares sont ainsi les fidèles qui ont commencé à s'intéresser à l'Afrique lorsqu'ils ont rejoint un groupe de culte " akan ». Bien au contraire, leur adhésion au mouvement vient en général compléter une entreprise de retour à ce qu'ils considéraient comme leur identité ancestrale, déjà amorcée depuis plusieurs années. L'adepte considère alors qu'en pratiquant la religion « akan », il s'attaque au fondement de cette culture africaine tant convoitée : la spiritualité.

15 Notons que les noms donnés par Dinizulu à sa maison de culte et à son centre culturel sont tous les deux constitués de termes twi, ga et anglais. En effet, l'expression Bosum Dzemawodzi est un amalgame du terme « divinité » en twi (bosum ou obosom) et de la traduction ga de ce même terme (dzemawodzi). L'appellation The Aims of the Modzawe, quant à elle, fait référence au lieu de réunion des aînés dans un village ga. 
En général, l'adepte potentiel participe à sa première cérémonie « akan » accompagné d'une connaissance, ami ou membre de la famille, qui lui-même appartient à l'une des maisons de culte du pays. Il est accueilli par les akomfo et on lui explique certaines des croyances partagées par les fidèles. Ainsi, chaque rituel organisé à New York par l'association The children of Akonnedi débute par un discours introductif au cours duquel Nana Baakan Yirenkiwah ou Nana Kodjo Ayesu, deux officiants du groupe, précisent à l'assistance la nature des divinités qui seront vénérées ce jour ainsi que les raisons pour lesquelles sont versées des libations. En général, les officiants expliquent également à l'assistance les phénomènes de transe rituelle dont ils seront les témoins pendant la cérémonie. A Washington D.C., Nana Kyerewaa demande à ses akomfowa (novices) de faire circuler, parmi l'ensemble des participants, des documents dans lesquels les attributs des divinités sont détaillés et certains des rituels explicités.

S'il éprouve de l'intérêt pour la cérémonie à laquelle il a assisté, l'adepte potentiel est invité par la personne qui l'avait accompagné au rituel dans sa maison de culte ${ }^{16}$. Là, il fait officiellement la connaissance du chef de culte qui effectue pour lui une séance de divination visant à le «présenter » (introduce to) aux abosom. Lors de cette première divination, la personne donne des offrandes au Nana qui l'accueille et aux abosom qui lui « ouvrent leur porte ».

A partir de cette première rencontre, l'adepte continue à rendre régulièrement visite au Nana ainsi qu'à participer à certains des rituels organisés dans la maison de culte. Progressivement, il est considéré, par les adeptes, comme l'un des leurs, et le chef de culte consent à symboliser son appartenance à la maison par une série de rituels qui feront de lui un réel «Akan » américain. Une fois ces rituels exécutés, l'intéressé devient un adepte reconnu de tous et peut se revendiquer comme le représentant d'une tradition " akan » jugée ancestrale et transmise à l'intérieur d'une généalogie d'officiants à laquelle il a été inclus.

Il est quatre rituels qui affirment l'appartenance du nouvel adepte à une maison de culte. Selon les maisons de culte mais aussi selon les fidèles concernés, ces quatre rituels sont effectués dans un ordre différent et sur une période plus ou moins longue. Le plus souvent, toutefois, le nouveau fidèle reçoit en premier lieu un nom rituel (shrine name) qui deviendra son identité principale au sein de sa famille religieuse. En vue de ce rituel de dation du nom, le chef de culte demande à l'adepte de venir le consulter pour une séance de divination. Lors de ce rituel, comme pour chaque divination, le prêtre se place sur son trône, à côté du client, devant l'autel de sa divinité protectrice. Il agite d'abord la cloche habituellement utilisée pour attirer l'attention des abosom, verse des libations, puis interroge directement la divinité en présence sur la supposée identité africaine de l'adepte. Grâce à ses dons de médium, le Nana est à même d'entendre la réponse de la divinité, qui parfois apporte également des précisions sur la signification de l'appellation alors révélée.

Les noms attribués aux «Akan » américains lors de ces séances de divination sont composés de deux particules. La première est relative au jour de naissance de l'adepte ainsi qu'à son sexe. Un homme né un lundi, par exemple, se verra décerner le nom de Kodjo

16 En général, la première cérémonie à laquelle assiste l'adepte potentiel n'a pas lieu à l'intérieur de la maison de culte mais dans un local pouvant accueillir un plus grand nombre de participants. Les futurs adeptes découvrent donc souvent la religion « akan » lors de cérémonies publiques, tel l'Akwasidae, organisées sur la base d'un calendrier rituel précis. 
alors qu'une femme née le même jour sera appelée Adua. La seconde est constituée de noms propres aux réseaux de parenté de Nana Oparebea, en particulier à son clan : Asona. Ainsi, Agyiriwah, Tacheampong, Agebari, Mantiebia, Opare, Korantemaa constituent des appellations très courantes au sein du mouvement « akan ».

Du fait de ce nom akan, chaque membre du mouvement peut se revendiquer comme un héritier direct de Nana Oparebea. Toutefois, plus que des descendants de ces lignages akan en Amérique, les adeptes états-uniens ont plutôt tendance à se décrire comme les représentants, voire les réincarnations, de la personne dont ils portent le nom. Dans cette logique, les Agyiriwah et les Tacheampong, en général des femmes, se revendiquent comme les incarnations modernes des sœurs d'Oparebea alors que les Korantemaa s'associent à une ancienne officiante de l'Akonedi Shrine, liée elle aussi à la prêtresse en chef par son réseau de parenté. De leur côté, les Agebari et les Opare célèbrent l'héritage des frères de la prêtresse alors que les Boateng, seule appellation non Asona décernée par Oparebea, symbolisent l'époux ashanti de la chef de culte à l'intérieur du mouvement américain.

Le rituel de dation du nom akan est suivi d'un second rite lors duquel le ou la Nana du lieu exécute sur le bras droit du fidèle des scarifications constituées d'une ligne verticale dont partent sept branches en diagonale. A côté de cet insigne, se cicatrisent sur le bras de l'adepte sept pointillés, dessinés eux aussi en diagonale. A l'Akonedi Shrine de Larteh Kubease, de semblables scarifications sont effectuées lors de la cérémonie marquant la fin du périple initiatique du nouveau prêtre (Opoku 1978). Aux Etats-Unis, ces scarifications sont pourtant exécutées plus tôt dans le parcours spirituel du fidèle et revêtent, pour les adeptes, une signification bien différente.

En effet, sur le modèle de l'arbre généalogique, les «Akan » américains donnent à cet ensemble de scarifications le nom d' « arbre » (tree). Il s'agit, pour eux, du symbole de leur appartenance à toute une généalogie d'officiants, qui, descendants de l'Akonedi Shrine de Larteh Kubease, pratiquent la religion « akan » en Afrique comme aux Etats-Unis. Le tree, devient ainsi l'emblème même de cette religion qui lie les "Akan » américains à leurs " pairs » ghanéens dans le cadre d'une communauté transnationale d'adeptes. Les fidèles portent, dans leur chair même, les signes de leur appartenance au mouvement américain d'une part, ainsi qu'à une tradition censée faire d'eux des Africains pouvant se revendiquer d'une maison-mère ghanéenne d'autre part. Ainsi, le tree vient compléter symboliquement le rituel de la dation du nom. Si le premier incluait l'Afro-Américain dans le clan d'Oparebea, le second fait de lui l'un de ses enfants spirituels, un membre de sa famille de religion. Ce faisant, le fidèle peut alors se revendiquer comme doublement «Akan ». Il est à la fois un supposé membre du lignage « biologique » d'Oparebea et son « fils » ou sa « fille» spirituel(le).

Après la cérémonie des scarifications, le ou la Nana de la maison de culte donne à l'adepte un bain spirituel (spiritual bath) destiné à préparer son corps à une éventuelle possession par les divinités. Il s'agit ici, grâce à un liquide concocté par le prêtre, de purifier le corps de l'adepte afin qu'aucun esprit négatif ne vienne compromettre sa connexion avec les entités et que son corps soit ouvert (opened) pour une communication optimale avec celles-ci. On considère que l'"Akan » américain est ainsi purifié des méfaits de la société occidentale sur son être et qu'il va pouvoir laisser libre cours à sa « personnalité africaine ».

Purifié, prêt à être possédé par les divinités sans que sa santé n'en soit menacée, l'«Akan » américain se voit alors remettre les colliers rituels, ahene (sing. ohene), 
symbolisant chacune des divinités vénérées par les pratiquants états-uniens. Ainsi, il reçoit l'ohene rouge et blanc symbolisant la divinité Asuo Gyebi tout comme celui qui, ocre, blanc et noir est associé à Nana Esi. Pour les «Akan » américains, ces colliers placent l'adepte sous la protection des abosom et rendent visibles à tout un chacun les liens que celui-ci a déjà établis avec les divinités. Arborant ses colliers et son tree, portant un nom akan et ayant reçu un bain spirituel, le membre du mouvement affirme son identité « akan » et peut potentiellement être appelé par les abosom au service de leur culte.

Ainsi purifié et « akanisé », le fidèle, s'il est l'objet de transes rituelles, devra alors se soumettre à un périple initiatique d'une durée moyenne de trois ans au cours duquel il apprendra à orchestrer les rituels et à " posséder » correctement les entités ${ }^{17}$. Pour les «Akan » américains, c'est alors la possession qui constitue l'aspect le plus important de leur apprentissage. Lors de nombreuses cérémonies, ils apprennent à incarner correctement les abosom, à effectuer les pas de danse qui leur sont associés et à adopter leurs postures et attitudes. En réalité, tout se passe comme si la possession constituait un moyen privilégié pour refaire corps avec leur être « akan ». En incorporant les abosom, ils considèrent ainsi qu'ils réactivent cette africanité enfouie au plus profond de leur être. Grâce à la maîtrise de leur corps purifié, ils deviennent des intermédiaires privilégiés entre les Afro-Américains et leur ancestralité africaine, des intermédiaires entre leur « passé » africain ou « akan » et leur présent afro-américain.

Officiants du mouvement « akan », les fidèles se revendiquent alors membres d'une ethnie akan transnationale ${ }^{18}$. Leur adhésion à un groupe de culte les a fait passer du statut d'African-American ${ }^{19}$ à celui d'Akan, exilés aux Etats-Unis. Ils ne se revendiquent plus comme des citoyens américains mais comme les membres d'une ethnie existant à cheval entre deux continents, se déployant par le truchement de réseaux transnationaux. L'identité américaine est ainsi niée et d'une certaine manière, l'identité raciale Black est remplacée par une identification ethnique symbolisée par l'utilisation du terme « akan ». Toutefois, l'observation attentive des rituels permet de mettre en avant d'autres niveaux d'identification, d'autres espaces du rituel et d'autres termes mobilisés pour définir son identité et recréer son passé.

\section{Etre "Akan ", c'est être panafricain}

Pour les membres du mouvement « akan », le périple initiatique, la quête spirituelle, sont indissociables d'un processus de retour aux origines. Utilisant plusieurs médiums pour se documenter, allant de la consultation de sites internet à la lecture d'ouvrages spécialisés rédigés tant par des anthropologues que des historiens ${ }^{20}$, l'adepte n'hésitera pas

17 Les «Akan » américains utilisent l'expression « posséder les abosom ». ce faisant, ils se considèrent comme des agents dans l'acte de posséder, action qui leur permet de refaire volontairement corps avec leurs origines.

18 Sur cette notion, voir Guedj (2004).

19 Le terme African-American est aujourd'hui majoritairement utilisé aux Etats-Unis pour désigner les populations noires. Il s'agit d'un terme à connotation idéologique qui insiste sur la composante africaine de l'identité noire américaine.

20 Les fidèles du mouvement « yoruba » utilisent également la littérature anthropologique pour assouvir leur soif de connaissance de l'Afrique. Le sort fait à ces écrits au sein du mouvement a été analysé par Kamari Clarke (2004). 
non plus à puiser des informations au sein d'autres modalités de culte dites « africaines » présentes sur le territoire américain.

En effet, à côté du mouvement « akan », se sont implantés aux Etats-Unis, à partir des années 1960, d'autres groupes de culte revendiquant eux aussi leur ancestralité africaine. La religion « yoruba » américaine tout d'abord, s'est constituée autour de la revendication par certains Afro-Américains d'un héritage des Yoruba du Nigeria. Elle est née du processus de réafricanisation de la santería cubaine, religion apparue aux EtatsUnis, suite à la migration massive de Cubains dans le pays ${ }^{21}$. Le mouvement kemet, quant à lui, prône l'adoption pour les Noirs de pratiques jugées originaires de l'Egypte antique. Il se déploie en deux associations : l'Ausar Auset Society, fondée par Ra Un Nefer Amen I en 1973 et la Smai Tawi Ankh Ascension Renaissance créée à Brooklyn dans les années 1980. A présent, ces modalités de culte coexistent dans les villes américaines, regroupées par les fidèles sous l'appellation African Traditional Religions (ATR) et formant un ensemble « africain » à l'intérieur du champ religieux afro-américain.

Aujourd'hui, les African Traditional Religions constituent un vaste ensemble au sein duquel les fidèles circulent, passant d'une modalité de culte à une autre et cumulant les initiations. Investis dans une réelle quête spirituelle, les adeptes usent de plates-formes pour échanger leurs points de vue sur les différents cultes en présence et confronter leurs expériences. A Philadelphie, l'Odunde Festival2 ${ }^{22}$, procession organisée tous les ans sous l'égide de la divinité yoruba Oshun, est devenu un lieu de réunion où se rencontrent pratiquants des religions kemet, " akan » et " yoruba ». Sur internet, le site d'Assata Shakur, ancienne membre du Black Panther Party et aujourd'hui pratiquante de la religion kemet, permet également aux fidèles de se donner des conseils et de commenter leurs « parcours spirituels ». On y retrouve les témoignages d'Afro-Américains amorçant leur cheminement religieux et souvent avides d'informations sur plusieurs cultes :

\begin{abstract}
Je ne me suis pas encore fixé sur une religion, écrit Ntangutizi, 'The Die Hard Afrikan'. J'ai rencontré des membres de l'Ausar Auset Society et de la Smaï Tawi Ankh Ascension Renaissance. Ils m'ont vraiment impressionné. Je suis allé à une cérémonie akan organisée par Onipa Abusia ${ }^{23}$ dans le Queens. J'étudie les traditions yoruba à travers des livres comme le Handbook on Yoruba Religious Concepts. Je lis aussi beaucoup sur l'histoire de Kemet et m'inspire des enseignements de Cheikh Anta Diop, Dr. Ben et Ra Un Nefer Amen ${ }^{24}$. Pour l'instant, j'explore. Cet été, je vais m'installer à Brooklyn et compte à cette occasion m'investir plus sérieusement dans un ou plusieurs de ces groupes (http://www.assatashakur.org/ forum/traditional/, posté le 02/01/2008, consulté le 20/01/2008).
\end{abstract}

21 Voir, entre autres, Capone (2005) et Clarke (2004).

22 Sur l'Odunde Festival, voir Guedj (2006).

23 Onipa Abusia est une maison de culte « akan » fondée en 1997 par deux des coépouses de Nana Yao Opare Dinizulu.

24 Cheikh Anta Diop, Yosef Ben-Jochannan, dit Dr. Ben, et Ra Un Nefer Amen sont tous les trois associés à la valorisation de l'héritage africain des Afro-Américains à travers le prisme de l'Egypte. Les écrits de Diop sur l'unité culturelle d'une Afrique noire puisant sa source en Egypte sont amplement cités par les Afrocentristes. Ben-Jochannan, activiste afro-américain s'est illustré par ses réflexions sur les prétendues origines égyptiennes du Christianisme. Enfin Ra Un Nefer Amen, ancien membre du mouvement « yoruba », est le fondateur de l'Ausar Auset Society, aujourd'hui principale organisation kemet du pays. 
A l'image de Ntangutizi, au sein du mouvement « akan », nombre de fidèles cumulent dans leurs «parcours spirituels » des affiliations à plusieurs groupes. Ainsi, Nana Baakan, une de mes principales informatrices au sein du mouvement, a quitté l'Eglise baptiste qu'elle fréquentait dans les années 1960 pour la Nation of Islam, lorsqu'elle est entrée à l'université. Adhérant à ce mouvement, elle mit fin à ses études, épousa un disciple de Fard Muhammad, fondateur du mouvement, et se consacra à la prédication « musulmane » dans les rues de Philadelphie. Plus tard, à la mort d'Elijah Muhammad en 1975, elle suivit le rénovateur du mouvement, Wallace D. Muhammad, au sein de la nouvelle organisation qu'il venait de créer : la World Community of Islam in the West, groupe dit « orthodoxe » et sunnite. Trois ans plus tard, la jeune femme décida de renoncer à l'islam pour intégrer un groupe de culte dit " africain », satisfaisant davantage son entreprise de quête des origines. Après un passage au sein d'un temple kemet, elle rejoignit une maison de culte « yoruba », puis devint prêtresse de la religion « akan ».

Aujourd'hui, malgré le caractère accidenté de son parcours spirituel, Nana Baakan considère que chacune de ces étapes était nécessaire pour comprendre la mission que les dieux akan lui ont assignée. Bien que la prêtresse reconnaisse la complémentarité des African Traditional Religions, elle prétend qu'il n'y aura pas de nouvelles « transitions » dans son existence et qu'elle restera toujours une «Akan ». En effet, pour la prêtresse, si son initiation à la religion « akan » constitue le parachèvement de son chemin spirituel, c'est parce que ce culte serait celui qui lui aurait permis de faire le lien entre plusieurs de ses expériences spirituelles passées. Le culte " akan » serait, selon elle, " inclusif », c'est-à-dire qu'il serait à même de digérer des pratiques empruntées à plusieurs systèmes de croyance, d'adopter en son sein des divinités étrangères qui, mêlées aux abosom, « acquerraient leur sens réel» (would make sense) $)^{25}$.

Le cas de Nana Baakan est loin d'être exceptionnel parmi les pratiquants des ATRs, notamment parce que c'est avec les entités yoruba (les orisha), qu'outre les abosom, elle entretient les relations les plus étroites. J'ai en effet, au cours de mes recherches aux EtatsUnis, rencontré à maintes reprises des prêtres initiés à la fois dans les systèmes « yoruba » et « akan », et des fidèles « yoruba » américains s'intéressant voire s'initiant aux pratiques ghanéennes. En dépit des nombreuses formes d'accusations que se portent les deux mouvements, il semble que, pour ces adeptes-là, ce soit dans leur association que les religions « yoruba » et « akan » révèlent leur plus grande efficacité. Le cumul d'initiations devient pour le fidèle une source de prestige, un signe de maturité et d'accomplissement de son parcours spirituel.

La maison de culte dirigée par Nana Baakan à Philadelphie accueille régulièrement des adeptes initiés dans la religion "yoruba ». Ceux-ci participent aux rituels et sont présents lors de toutes les célébrations orchestrées par Nana Baakan. Parmi eux, Sangotola et Ogunfumilayo, deux prêtresses aux noms yoruba, sont particulièrement actives. Initiées au Nigeria et au Ghana, elles font figure d'autorité et lient, dans leur quotidien, pratiques « yoruba » et « akan ».

Chez elles, à Baltimore, Sangotola et Ogunfumilayo adoptent alors une série d'attitudes jugées spécifiquement « akan ». Lorsqu'elles entrent dans leur habitation commune, les deux femmes ôtent leurs chaussures devant la porte, ce qu'elles font systématiquement

25 Ces informations sur le parcours spirituel de Nana Baakan ont été recueillies lors d'une série d'entretiens réalisés à Philadelphie en juin 2002, mars 2003, 2004, 2005 et août 2007. 
lorsqu'elles se rendent chez Nana Baakan. Quand elles reçoivent un visiteur, elles le font patienter dans la salle de séjour avant de lui donner un verre d'eau porté sur un plateau, de lui demander sa " mission », et finalement de lui souhaiter la bienvenue dans leur demeure. Comme tous les membres du mouvement « akan », elles rassemblent les offrandes de leurs hôtes dans un saladier qu'elles montent sur leurs têtes dans la chambre des autels. Dans cette chambre des autels, Sangotola et Ogunfumilayo ont installé deux représentations pour les abosom et les orisha. Le matin, elles y versent des libations de gin, pratique « akan », en prononçant des incantations yoruba. Puis, elles usent de leurs dons de médium pour communiquer avec chacune de ces divinités en anglais (photo 1$)^{26}$.

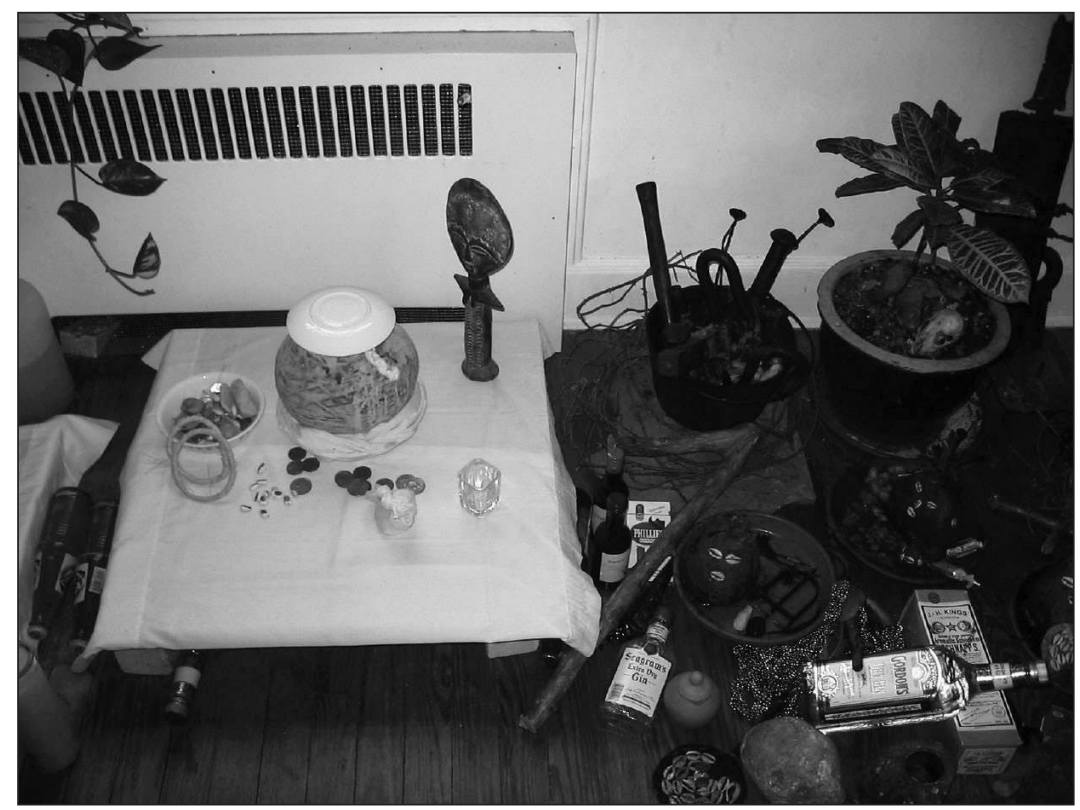

Photo 1

A Baltimore, dans la chambre des autels d'Ogunfumilayo et de Sangotola, un autel (à gauche) consacré à la divinité akan Adade Kofi côtoie celui de l'orisha Ogún (à droite). Devant, des représentations des Eshu, des bouteilles de gin et de schnaps, «boisson préférée des abosom ».

(C) Pauline Guedj.

Dans leur vie quotidienne, Sangotola et Ogunfumilayo ont donc instauré une série d'équivalences entre les systèmes "yoruba » et " akan ", équivalences se manifestant aussi bien dans le rituel que dans la caractérisation des entités spirituelles. En effet, pour les fidèles de la maison de culte dirigée par Nana Baakan, les « abosom et les orisha travaillent ensemble ${ }^{27}$. Chaque orisha trouve son équivalent dans le monde « akan » alors que chaque abosom peut être associé à une divinité yoruba. Ainsi, Asuo Gyebi, l'une des principales divinités akan, est souvent associée au Shangó yoruba, partageant

26 Informations recueillies lors d'enquêtes de terrain effectuées à Baltimore en 2004-2005.

27 Cette expression a été prononcée par Nana Baakan lors d'un entretien réalisé à Philadelphie en octobre 2004. 
avec celui-ci son arrogance et sa dignité, alors qu'Ogún devient, pour les «Akan », la version yoruba d'Adade Kofi, obosom autoritaire lié au fer. Lors des cérémonies rituelles, les «Akan » américains sont à même d'incarner par la transe chacune de ces divinités mettant en scène des abosom et des orisha dansant, chantant et conversant ensemble.

En réalité, tout se passe ici comme si les fidèles « akan » cherchaient à forger aux Etats-Unis une nouvelle religion, une religion qu'ils qualifient de « panafricaine ». Ainsi, si le processus de retour aux origines au sein du mouvement supposait la revendication d'origines ethniques akan, celui-ci traverse aujourd'hui une nouvelle phase qui s'appuie sur l'alliance de plusieurs systèmes de croyance, « yoruba » et « akan » en particulier, chacun jugé comme authentiquement africain. Religion panafricaine, le culte nouvellement créé s'appuie alors sur une conception de l'Afrique comme réceptacle d'une culture unique, originellement unifiée et dont les actuelles divisions seraient les produits du colonialisme et de l'intervention des Blancs ${ }^{28}$. Pour les «Akan » américains, construire une religion pensée comme panafricaine revient donc à se revendiquer comme les détenteurs d'un culte ancestral, revitalisé aux Etats-Unis, bien que disparu du continent noir.

Ce faisant, les membres du mouvement « akan » mobilisent une nouvelle catégorie d'identification pour mener à bien leur entreprise de retour aux sources. "Akan », reliés à des officiants ghanéens par une série de réseaux transnationaux (Guedj 2006 et 2008), ils sont également des panafricains, garants de la reproduction aux Etats-Unis d'une religion unifiée perçue comme originelle et première. Ainsi, les «Akan » américains considèrent que c'est la nature même de leur religion qui fait d'eux les détenteurs potentiels de cette «Pan-Afrique ». Le caractère « inclusif» de la religion « akan » porterait en lui les germes d'une religion panafricaine faisant des «Akan » américains des individus essentiellement panafricains. En d'autres termes, ce serait parce qu'ils pratiquent la religion « akan » aux Etats-Unis que les membres du groupe sont à même de se revendiquer à la fois comme des Akan, liés à un groupe ethnique d'Afrique de l'Ouest et comme des panafricains, dignes héritiers d'une Afrique originelle, pensée comme culturellement unie.

\section{Etre «Akan ", c'est aussi être Afro-Américain}

Les divinités et les rituels constituent donc un élément capital dans l'élaboration de l'identité « akan » aux Etats-Unis. Entretenant, dans les rituels, des relations avec d'autres divinités africaines, les orisha surtout, les abosom deviennent la preuve incarnée du penchant panafricain de l'identité « akan », penchant panafricain qui relie les adeptes à une Afrique originelle, saisie dans sa forme précoloniale. Toutefois, rarement vénérées seules, ces divinités sont également associées à d'autres entités, qui donnent à la pratique de cette religion aux Etats-Unis une coloration proprement afro-américaine. Alliés aux ancêtres afro-américains de leurs adeptes, les abosom et les orisha constituent ainsi un vaste ensemble d'entités spirituelles nécessitant des rituels communs qui permettront à l'«Akan » américain d'inclure ses propres expériences états-uniennes dans son entreprise de retour à l'Afrique.

Dans chaque maison « akan » se trouve ainsi une série d'autels consacrés au culte des ancêtres. Ceux-ci sont placés sur des tables hautes. Ils sont constitués de photos, de bibelots, de souvenirs, et sont souvent recouverts de tentures. Colorés et encombrés, ils

28 Cette idée d'une Afrique culturellement unie se retrouve dans les écrits de Cheikh Anta Diop et de Molefi Asante. Voir en particulier Asante et Asante (1985) et Diop (1959). 
contrastent fortement avec les autels dédiés aux abosom, situés, eux, à même le sol et composés uniquement d'un pot en terre cuite et d'une petite statuette en bois. Dans la maison de culte de Nana Baakan à Philadelphie, les autels pour les ancêtres sont séparés de ceux des divinités ghanéennes par un rideau. Ancêtres, abosom et orisha ne partagent donc pas le même espace, comme si les premiers constituaient une catégorie d'entités à part, à ne pas confondre avec les divinités africaines.

En réalité, lorsque Nana Oparebea enseigna à ses fidèles certaines des traditions religieuses akan, elle ne leur transmit pas les connaissances nécessaires, les symboles et les outils qui leur auraient permis de mettre en place dans le Nouveau Monde un culte des ancêtres semblable à celui que l'on rencontre à Larteh (Guedj 2006). A Larteh Kubease, le culte des ancêtres est en effet placé sous la responsabilité de l'autorité politique de la ville, le Kubeasehene. C'est lui, qui lors de rituels réguliers comme l'akwasidae, verse le sang des sacrifices sur des trônes «noircis » (blackened stools) symbolisant le pouvoir des ancêtres lignagers. Or aux Etats-Unis, bien que Dinizulu ait été honoré du titre de « Chef des Akan d'Amérique ", aucune maison de culte ne possède de trône noirci permettant la diffusion du culte des ancêtres. Partis au Ghana à la recherche d'ancestralité, les AfroAméricains membres du mouvement « akan » n’y ont en réalité trouvé que des divinités, dont l'énergie serait aujourd'hui contenue dans leurs autels. Détaché d'une affiliation ghanéenne, le culte des ancêtres tel qu'il est pratiqué aux Etats-Unis constitue alors pour les Afro-Américains « akan », un terrain d'innovation où ils purent élaborer un troisième espace rituel qui se développa en interaction avec celui habité par les abosom et les orisha.

Il est deux principaux types d'ancêtres représentés sur les autels. Dans la maison de culte de Nana Baakan se trouve ainsi une table présentant les ancêtres de la famille proche de la prêtresse, ses grands-parents, arrière- et arrière-arrière-grands-parents. L'usage de cet autel lui est réservé et les ancêtres qui y sont vénérés ne la possèdent que très rarement durant les transes rituelles. Le second autel est situé à gauche du premier. Guéridon recouvert de draps violet et blanc, l'autel est composé d'un saladier en porcelaine, d'une sculpture en bois représentant l'ankh, symbole égyptien de la clef de $v^{29}{ }^{29}$, de plusieurs bouteilles de Southern Comfort ${ }^{30}$, d'une pipe, et de deux cannes en bois sculptées. A côté de l'autel, se trouve une chaise à bascule (rocking-chair) d'où pend un chapeau de paille (photo 2).

Selon les propos de Nana Baakan, ce second autel est consacré à des entités qui sont venues la visiter dans ses rêves et lui ont demandé d'installer un emplacement sacré pour eux. Parmi ces entités qu'elle désigne tous à l'aide du terme anglais ancestors, Nana Baakan prend soin d'un ensemble de personnalités afro-américaines dont Martha, une femme qui progressivement lui aurait révélé la totalité de son histoire. Martha serait une femme originaire du Ghana qui, après un passage au Brésil, aurait été réduite en esclavage en Caroline du Sud. Là, elle serait devenue l'esclave préférée de son maître qui abusa d'elle à plusieurs reprises et lui donna dix enfants. Un jour, l'une des petitesfilles de Martha, la plus jeune, refusa de contenter les désirs du planteur. Furieux, celuici se vengea sur la grand-mère en lui crevant les yeux. Martha demeura ainsi, aveugle,

29 La présence sur cet autel de l'ankh est à mettre en relation avec l'affiliation passée de Nana Baakan à l'Ausar Auset Society. Ce symbole se retrouve toutefois fréquemment aussi bien sur les autels des «Akan » américains que sur les couvertures des ouvrages des Afrocentristes. 
dans la plantation pendant de longues années, où elle développa progressivement des talents de médium. Un jour, sa petite-fille eut raison du maître, mettant fin à des années de soumission servile.

Lorsqu'elle évoque Martha, Nana Baakan parle avec enthousiasme, multipliant les détails et les précisions sur la vie de cette femme qu'elle admire. Lors d'un entretien, elle me racontait ainsi les révélations de Martha, ancêtre qui lui rend visite dans ses rêves :

Martha était l'esclave préférée du maître. A chaque fois qu'il voulait coucher avec une des esclaves, il envoyait chercher Martha. Il la violait tout le temps, continuellement. Martha était très noire. Elle a vécu plus de cent ans. C'est quand elle est devenue aveugle qu'elle a commencé à être spirituelle. Elle portait un chapeau pour couvrir ses yeux, comme celui que j'ai sur son autel en bas. On ne pouvait rien faire en cachette quand elle était là parce que Martha voyait tout. Elle était un peu comme la Nana de la plantation, celle que tout le monde respecte et dont tout le monde a peur (Entretien avec Nana Baakan Agyiriwah, Philadelphie, juillet 2004).

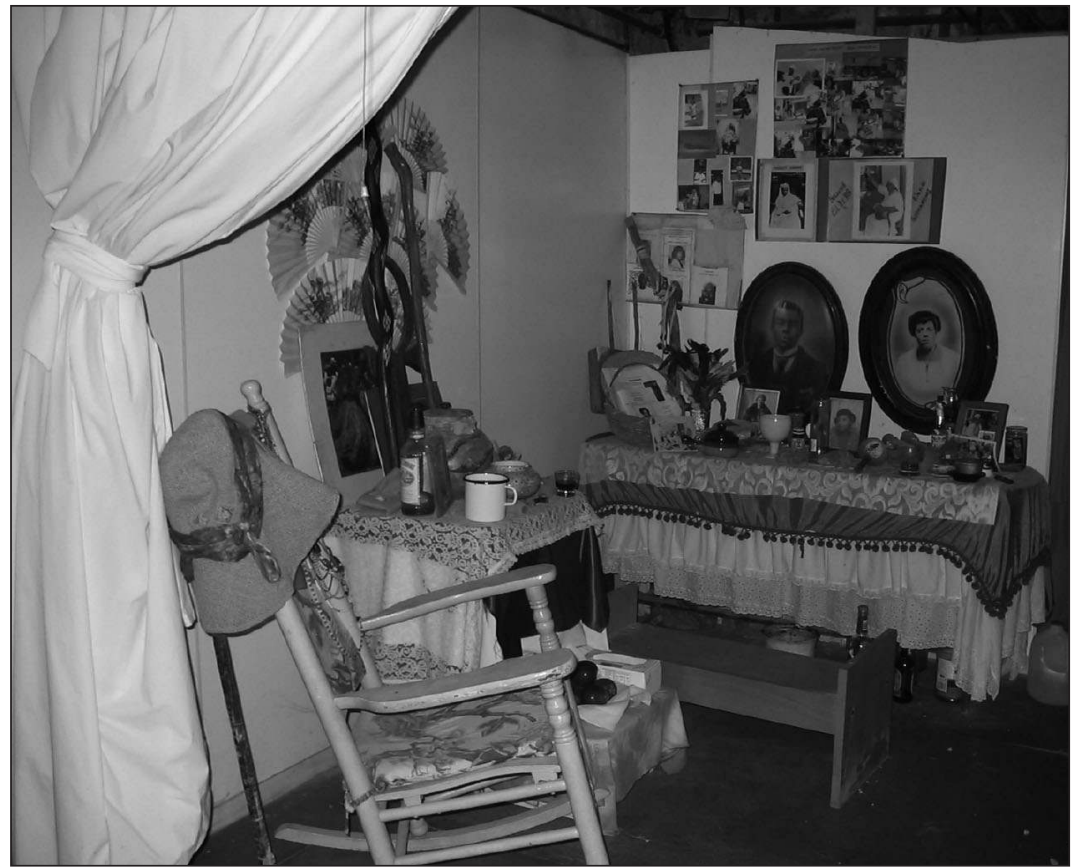

Photo 2

A Philadelphie, la maison de culte dirigée par Nana Baakan, l'Adade Kofi Sankofa Bosomfie, dispose d'un espace où sont rassemblés les autels des ancêtres. Au fond, se trouve l'autel des ancêtres familiaux de la prêtresse. On y aperçoit des photographies et un ensemble de bibelots leur ayant appartenus.

A gauche, l'autel de Martha avec sa chaise à bascule et son chapeau. (C) Pauline Guedj.

$\mathrm{Au}$ sein de cette maison de culte, Martha constitue, sans aucun doute, l'une des entités spirituelles les plus appréciées. Lors de mes recherches sur le terrain, les fidèles 
me racontaient fréquemment les cérémonies au cours desquelles Martha possède Nana Baakan et tient audience pendant plusieurs heures, revenant sur les détails de sa vie passée. Pour ces «Akan » américains, les récits de Martha sont toujours passionnants. Captivés, ils n'hésitent d'ailleurs pas à poser à l'ancêtre incarnée des questions précises afin d' " apprendre à la connaître ». Nana Baakan dispose, chez elle, de nombreux carnets sur lesquels l'une des akomfo de la maison, Nyo, a pris en note les interventions de l'ancêtre lors des cérémonies. Régulièrement, Nana use de ces carnets pour se remémorer certains épisodes de l'histoire personnelle de Martha.

Toutefois, si Martha connaît une telle popularité au sein de la maison de culte, c'est avant tout parce que, dans sa vie passée, elle a été victime de l'esclavage. D'une certaine manière, c'est toute l' « expérience » afro-américaine que Martha incarne dans la maison de culte, tout le passé douloureux de la communauté. Le récit de sa vie, telle que Baakan le transmet à ses adeptes, constitue alors un condensé des stéréotypes associés à la vie d'esclave dans les mentalités afro-américaines. Martha était une esclave de maison. Elle avait un statut particulier au sein de la plantation, ce qui parfois créait des conflits entre elle et les autres esclaves. Violée perpétuellement par le maître, elle avait enfanté une progéniture métisse au teint pâle dont elle ne pouvait apprécier la couleur de peau. La nuit, lorsqu'elle cherchait à échapper à la vie de la plantation, Martha s'asseyait sous le perron de la maison coloniale de son maître, sur une chaise à bascule, identique à celle que Nana Baakan a placé à côté de son autel. Coiffée de son chapeau de paille, la Bible sur les genoux, elle y fredonnait des negro spirituals et priait pour l'émancipation de son peuple.

Cette vision de Martha, seule, chantant des negro spirituals sur son rocking-chair constitue une réelle " image d'Epinal » de la vie du Sud avant la guerre de sécession. Martha est, pour les adeptes de la maison de culte, une figure emblématique, une héroïne, qui dans la plus grande douleur, a su attendre la décrépitude de son maître et a concouru, grâce à ses prières et à son espoir jamais perdu, à la libération de sa communauté. Ainsi, elle devient l'incarnation rêvée de ces ancêtres afro-américains réduits en esclavage dont les adeptes ne disposent souvent d'aucune trace, et qu'à côté des entités africaines, ils se proposent, à présent, de vénérer. Martha, ancêtre fédérateur au sein de la maison de culte, porte alors en elle le spectre de tous les arrière-grand-pères, toutes les grand-tantes des fidèles, tous ces hommes et ces femmes dont son histoire tragique permet de raviver la mémoire.

Aux côtés de Martha, nombreux sont les fidèles qui prétendent, eux aussi, avoir été « appelés » par des ancêtres victimes de l'esclavage. Ephraim, un jeune danseur âgé de 23 ans, me décrivait ainsi, lors d'une conversation, une ancêtre avec laquelle il entretient des relations particulièrement étroites. Prénommée Mary, celle-ci viendrait souvent lui rendre visite dans ses rêves et le posséderait fréquemment lors des cérémonies rituelles. Selon Ephraim, Mary est originaire du Togo. Agée d'une quinzaine d'années, elle fut déportée en Louisiane où elle fut transportée sur une plantation. Pour Ephraim, Mary est porteuse d'une énergie très difficile à contenir pendant les transes, comme si toute sa colère, les révoltes sur la plantation, restaient vivaces et ne demandaient qu'à s'exprimer lors des possessions.

Nyo, quant à elle, une femme d'une quarantaine d'années, me racontait lors d'un entretien effectué en juillet 2003, être en communication régulière avec une de ses ancêtres qui, elle aussi, a souffert indirectement de l'esclavage : Zafina. Originaire du 
Ghana, celle-ci fut témoin de l'enlèvement de son père et de sa mère contraints à partir en bateau vers les Amériques. Nyo prétend que Zafina assista, cachée derrière une dune de sable, à l'embarquement de sa famille pour le Nouveau Monde. Assise sur la plage, elle aurait alors regardé, en pleurant, le bateau s'éloigner vers l'Ouest.

Dans la maison de culte, lors de cérémonies appelées ancestors parties, il est alors fréquent que ces ancêtres s'incarnent en même temps et se remémorent les souvenirs tragiques de leur asservissement. Chaque membre de la maison de culte les connaît, sait les distinguer et est familiarisé avec les habitudes et les manies de chacun. En réalité, tout se passe comme si ces entités, toutes liées à une Histoire afro-américaine ou à l'expérience de l'esclavage, formaient une sorte de patrimoine que se partagent les membres du groupe.

Nous le voyons, aux Etats-Unis, dans les maisons de culte « akan », se trouveraient donc mêlés un culte consacré aux divinités africaines abosom et orisha, parfois même neteru de la religion kemet $^{31}$ et un culte voué aux ancêtres des pratiquants, afro-américains, ancêtres avec lesquels ne sont par ailleurs pas forcément entretenues des relations de filiation directe. A côté des divinités africaines, les ancêtres constituent une deuxième catégorie d'entités, propice à des adaptations afro-américaines du culte « akan » et à l'intégration de celui-ci au contexte états-unien. L'Afro-Américain pratiquant la religion « akan » vénère à la fois les symboles de ses origines africaines et de sa réalité afroaméricaine, elle-même réafricanisée.

En instaurant un culte des ancêtres dans leurs maisons de culte, c'est alors l'expérience traumatisante de l'esclavage, fondement de l'Histoire afro-américaine, que les adeptes cherchent à interpréter et à prendre en conscience. Là où la rhétorique de la plupart des mouvances politiques et nationalistes afro-américaines, la Nation of Islam par exemple, avait jusqu'à présent cherché à évincer l'esclavage pour revendiquer la continuité, l'absence de rupture entre l'Afrique et la « diaspora » noire, là ou Molefi Asante pouvait appeler les Afro-Américains à se tourner vers « la période qui a précédé l'esclavage » pour se « revitaliser », les membres du mouvement « akan » font de cet épisode tragique de leur Histoire leur particularité au sein de l'entité panafricaine, un «marqueur » de leur propre ethos. Il n'y a plus, pour eux, de contradiction entre une identité africaine recouvrée, qu'elle soit « akan » ou panafricaine et une conscience afro-américaine qui serait née de la perte de repères. Bien au contraire, leur « afro-américanité » devient « africanité » en même temps que leurs ancêtres sont vénérés par des processus rituels similaires à ceux réservés aux divinités « akan »: les prières en twi, les libations et la possession.

\section{Conclusion}

La diffusion de la religion « akan » aux Etats-Unis par le truchement de réseaux transnationaux s'est donc accompagnée de son indigénisation, de son intégration aux réalités du champ religieux local, et de son adaptation aux constructions identitaires ici à l'œuvre. Cette indigénisation a abouti à la fois à l'association du culte « akan » à celui d'autres divinités dites « africaines », mais aussi à la création à l'intérieur de celui-ci d'un espace rituel spécifiquement afro-américain. Se revendiquant membre d'une communauté transnationale « akan », le fidèle ne renonce pas pour autant à affirmer ses spécificités non

31 Nous avons vu qu'il existe également aux Etats-Unis un culte kemet. Dans certaines maisons « akan », les abosom sont alors associés aux divinités kemet, les neteru. 
seulement dans une entité panafricaine globale, mais aussi dans le contexte local des Etats-Unis.

Dans cet article, j'ai donc tenté de montrer comment l'identité « akan » américaine se construit en réalité autour de trois lignes directrices qui se tissent dans le discours et les pratiques des adeptes, formant une trame tantôt harmonieuse, tantôt discordante. En effet, d'un côté, les fidèles qui intègrent le mouvement renoncent à leur identité noire américaine pour adopter une identité nouvelle, « akan », perçue comme semblable à celle de leurs ancêtres réduits en esclavage et déportés dans le Nouveau Monde. Pour ce faire, ils se soumettent, nous l'avons vu, à plusieurs rituels dont le but est à la fois de les purifier des méfaits que la société blanche leur a fait subir mais aussi de leur accorder les signes d'appartenance à leur groupe d'adoption. Cette identité « akan », ils la mettent alors en scène lors des cérémonies de transe rituelle où " possédant » les abosom, ils incorporent pleinement leur akanité.

D'un autre côté, les adeptes du mouvement « akan » aspirent à une identité panafricaine qu'ils élaborent dans leurs parcours spirituels et religieux en cumulant les initiations et en se déclarant membres de plusieurs organisations dites « africaines ». A la fois «Yoruba » et «Akan », ils s'imaginent les descendants directs d'Africains pré-coloniaux qui, n'ayant pas connu le démantèlement de leur culture, n'auraient jamais souffert des barrières ethniques imposées par les pouvoirs occidentaux. Etre « panafricain » signifie alors, pour eux, être un Africain originel c'est-à-dire plus « authentique » que les habitants actuels du continent noir. Dans le rituel, cette construction d'une identité revendiquée clairement comme « panafricaine » se retrouve dans les aménagements entre religion « akan » et culte « yoruba ». Incorporer les abosom et les orisha ensemble, les vénérer dans des autels communs revient alors à affirmer ce sentiment d'appartenance " panafricain », sentiment d'appartenance qui serait validé par les divinités africaines elles-mêmes.

Enfin, les «Akan » américains se revendiquent comme les acteurs d'une « expérience afro-américaine» qui, elle aussi, constitue un "marqueur » de leur identité et qui les différencie à la fois des Noirs provenant des autres régions du Nouveau Monde et des Africains du continent. L'identité afro-américaine, à laquelle il fallait renoncer lorsque, intégrant le mouvement, on devenait « akan », devient aujourd'hui matière à revendication. Dans le rituel, c'est le culte des ancêtres qui constitue l'espace d'élaboration de ce troisième volet de l'identité « akan » américaine. Là où les "Akan » en quête de panafricanisme alliaient, dans un même culte, orisha et abosom, ils font désormais de leurs ancêtres afro-américains des équivalents des entités et des divinités africaines, qui, eux aussi, peuvent interagir avec elles et communiquer avec l'Afrique. Grâce au culte des ancêtres, les "Akan » américains font de leur " afro-américanité » une composante de leur « africanité ».

L'histoire du mouvement « akan », l'élaboration du discours de ses membres et des rituels consistent alors principalement en une négociation constante entre ces trois sentiments d'appartenance et ces trois catégories d'identification. Selon les circonstances, comme c'est souvent le cas lorsqu'on parle d'identité, les "Akan » américains se définissent tantôt comme des "Akan », tantôt comme des " panafricains » et tantôt comme des African-Americans. S'ils s'adressent à un membre du mouvement « yoruba » par exemple, c'est leur identité « akan » qu'ils valorisent. Si au contraire, ils participent à une réunion rassemblant des adeptes de diverses African Traditional Religions, ils insistent sur leur appartenance à une entité « panafricaine ». Enfin, s'ils sont confrontés 
à des Akan du Ghana qui critiquent leur entreprise de retour aux sources, ils insistent sur leurs caractéristiques afro-américaines.

En quête d'un passé, d'une mémoire dont ils cherchent à combler les «trous », les " Akan » américains s'inventent une identité multiple, où, pour reprendre les termes de Jean-Loup Amselle (2001), le « branchement » devient une logique permanente de fonctionnement. Pour exister, pour résister aux affres des troubles identitaires, on se « branche » alors successivement sur les Akan, le Ghana, les Yoruba, une « Pan-Afrique » imaginée comme culturellement unie et une expérience " afro-américaine » intégrant la tragédie de l'esclavage. Or, dans cette logique, seuls le culte et l'action rituelle parviennent à donner à cette identité fractionnée sa cohérence tant espérée.

\section{Références citées}

Amselle, Jean-Loup, 2001. Branchements : anthropologie de l'universalité des cultures. Paris : Flammarion.

Asante, Molefi, 2005. L'Afrocentricité et la résurgence africaine, colloque « Le rôle de l'afrocentrictité dans la renaissance africaine », 29 et 30 juillet, Cotonou, Bénin.

Disponible sur http://www.africamaat.com/L-Afrocentricite-et-la-Resurgence.

Asante, Molefi Kete et Kariamu Welsh Asante, 1990 [1985]. African Culture. The Rythms of Unity. Trenton : Africa World Press.

BAstide, Roger, 1996. «Continuité et discontinuité des sociétés et cultures afro-américaines », Bastidiana, (13-14), pp. 11-28.

BRENT Turner, Richard, 1984. Islam in the African American Experience. Bloomington et Indianapolis : Indiana Press University.

Brokensha, David W., 1966. Social Change at Larteh, Ghana. Oxford : Clarendon Press.

CAPOnE, Stefania, 2005. Les Yoruba du Nouveau Monde. Religion, ethnicité et nationalisme noir aux Etats-Unis. Paris : Karthala.

Clarke, Maxine Kamari, 2004. Mapping Yoruba Networks. Power and Agency in the Making of Transnational Communities. Durham et Londres : Duke University Press.

Diop, Cheikh Anta, 1959. L'unité culturelle de l'Afrique noire. Paris : Présence africaine.

Essien Udom, E.U, 1964 . Black nationalism : A search for Identity in America. New York : Dell publishing.

GARDEll, Mattias, 1996. In the Name of Elijah Muhammad : Louis Farrakhan and the Nation of Islam. Durham : Duke University Press.

Gilroy, Paul, 2003. L'Atlantique noir. Modernité et double conscience. Cahors : Editions Kargo.

GuEdJ, Pauline,

2004. «'A Nation within Nations' : nationalisme afro-américain et réafricanisation aux Etats-Unis », Civilisations, LI (1-2), pp. 23-38.

2005. « Du Panafricanisme à la réafricanisation : formation et actualités du mouvement akan aux EtatsUnis, Journal de la Société des Américanistes, 911, pp. 93-112.

2006. Le Chemin du Sankofa. Religion et Identité « akan » aux Etats-Unis, Thèse de Doctorat, Université Paris X Nanterre.

2007. « Entre Africanité et Afro-Américanité. Divinités « akan » et culte des ancêtres aux Etats-Unis », Ateliers du LESC, 31, http://ateliers.revues.org/document752.html.

2008. « De l'Afrique aux Amériques. L'implantation du culte des divinités akan aux Etats-Unis », Diasporas. Histoires et Sociétés, 12, pp. 85-100.

Halbwachs, Maurice, 2002 [1950]. La mémoire collective. Paris : Albin Michel. 


\section{Pauline Guedj}

Hobsbawm, Eric et Terence Ranger (éds), 2006. L'invention de la tradition. Paris : Editions Amsterdam.

Lenclud, Gérard, 1994. "Qu'est-ce qu'une tradition ? », in M. Détienne (éd.), Transcrire les mythologies. Tradition, écriture, historicité. Paris : Albin Michel.

Moses, Wilson Jeremiah, 1998. Afrotopia : The Roots of African American Popular History. Cambridge : Cambridge University Press

Opoku, Kofi Asare, 1978. West African Traditional religion. Accra : FEP.

Palmie, Stephan, 1995. «Against Syncretism. ‘Africanizing' and 'Cubanizing' Discourses in North American a Worship » in Richard Fardon (éd.) Counterworks. Managing the Diversity of Knowledge. New York et Londres : Routledge, pp. 73-104.

Poulllon, Jean, 1975. « Tradition : transmission ou reconstruction », in Jean Pouillon, Fétiches sans fétichisme. Paris : Maspéro.

Walker, Clarence, 2004. L'impossible retour. A propos de l'afrocentrisme. Paris : Karthala.

X, Malcolm, 1967. Malcolm X on Afro-American History. New York : Pathfinder Press. 